ISSN 1855-3966 (printed edn.), ISSN 1855-3974 (electronic edn.)

\author{
ARS MATHEMATICA CONTEMPORANEA 17 (2019) 271-275 \\ https://doi.org/10.26493/1855-3974.1901.987 \\ (Also available at http://amc-journal.eu)
}

\title{
Logarithms of a binomial series: A Stirling number approach
}

\author{
Helmut Prodinger \\ Department of Mathematics, University of Stellenbosch, \\ 7602, Stellenbosch, South Africa
}

Received 8 January 2019, accepted 21 April 2019, published online 15 October 2019

\begin{abstract}
The $p$-th power of the logarithm of the Catalan generating function is computed using the Stirling cycle numbers. Instead of Stirling numbers, one may write this generating function in terms of higher order harmonic numbers.
\end{abstract}

Keywords: Catalan numbers, logarithm, generating function, Stirling number.

Math. Subj. Class.: 05A15, 05A10

\section{Introduction}

Knuth [6, 7] proposed the exciting formula

$$
(\log C(z))^{2}=\sum_{n \geq 1}\left(\begin{array}{c}
2 n \\
n
\end{array}\right)\left(H_{2 n-1}-H_{n}\right) \frac{z^{n}}{n},
$$

where

$$
C(z)=\frac{1-\sqrt{1-4 z}}{2 z}=\sum_{n \geq 0} \frac{1}{n+1}\left(\begin{array}{c}
2 n \\
n
\end{array}\right) z^{n}
$$

and

$$
H_{n}=\sum_{1 \leq k \leq n} \frac{1}{k}
$$

with the generating function of Catalan numbers and harmonic numbers.

This formula was recently extended by Chu [1] to general exponents $p$. Chu's approach is based on the use of (exponential) Bell polynomials. Note that Knuth talked about the exponent 1 in his Christmas lecture from 2014 [5].

E-mail address: hproding@sun.ac.za (Helmut Prodinger) 
We present here a very simple approach to this question using Stirling cycle numbers; recall [3] that they transform falling powers into ordinary powers viz.

$$
x^{\underline{n}}=\sum_{0 \leq k \leq n}\left[\begin{array}{l}
n \\
k
\end{array}\right](-1)^{n-k} x^{k} .
$$

For the readers' convenience it is mentioned that the numbers $\left[\begin{array}{l}n \\ k\end{array}\right](-1)^{n-k}$ appear often in the older literature as $s(n, k)$ and are then denoted as Stirling numbers of the first kind.

\section{The expansion of the $p$-th power}

The substitution $z=\frac{u}{(1+u)^{2}}$ was presented in [2] and it is extremely useful when dealing with Catalan numbers and Catalan statistics. Using it in (1.1), we get $C(z)=1+u$, and, by the Lagrange inversion formula [8],

$$
u^{m}=\sum_{n \geq m} \frac{m}{n}\left(\begin{array}{c}
2 n \\
n-m
\end{array}\right) z^{n}
$$

for $m \geq 1$. For $m=0$ the formula is still true when taking a limit. We now consider the bivariate generating function

$$
\begin{aligned}
F(z, \alpha) & =\sum_{p \geq 0} \frac{\alpha^{p}}{p !}(\log C(z))^{p}=\exp (\alpha \log C(z)) \\
& =C^{\alpha}(z)=(1+u)^{\alpha}=\sum_{m \geq 0}\left(\begin{array}{c}
\alpha \\
m
\end{array}\right) u^{m}
\end{aligned}
$$

But

$$
\left(\begin{array}{c}
\alpha \\
m
\end{array}\right)=\frac{1}{m !} \alpha^{\underline{m}}=\frac{1}{m !} \sum_{0 \leq k \leq m}(-1)^{m-k}\left[\begin{array}{c}
m \\
k
\end{array}\right] \alpha^{k}
$$

Therefore

$$
F(z, \alpha)=\sum_{0 \leq k \leq m \leq n} \frac{1}{m !}(-1)^{m-k}\left[\begin{array}{c}
m \\
k
\end{array}\right] \alpha^{k} \frac{m}{n}\left(\begin{array}{c}
2 n \\
n-m
\end{array}\right) z^{n} .
$$

The desired formula follows from reading off coefficients of $\alpha^{p}$ :

$$
(\log C(z))^{p}=p !\left[\alpha^{p}\right] F(z, \alpha)=\sum_{p \leq m \leq n} \frac{p !}{m !}(-1)^{m-p}\left[\begin{array}{c}
m \\
p
\end{array}\right] \frac{m}{n}\left(\begin{array}{c}
2 n \\
n-m
\end{array}\right) z^{n} .
$$

\section{Special cases}

For $p=1$ in equation (2.1), we get the instance of the Christmas lecture:

$$
\log C(z)=\left[\alpha^{1}\right] F(z, \alpha)=\sum_{1 \leq m \leq n} \frac{1}{m !}(-1)^{m-1}\left[\begin{array}{c}
m \\
1
\end{array}\right] \frac{m}{n}\left(\begin{array}{c}
2 n \\
n-m
\end{array}\right) z^{n} .
$$

Since $\left[\begin{array}{c}m \\ 1\end{array}\right]=(m-1)$ !, this leads to

$$
\log C(z)=\left[\alpha^{1}\right] F(z, \alpha)=\frac{1}{2} \sum_{n \geq 1} \frac{1}{n}\left(\begin{array}{c}
2 n \\
n
\end{array}\right) z^{n} .
$$


Now we turn to the instance $p=2$ from $[6,7]$. (Note that $\left[\begin{array}{c}m \\ 2\end{array}\right]=(m-1) ! H_{m-1}$.) Equation (2.1) leads to

$$
\begin{aligned}
2\left[\alpha^{2}\right] F(z, \alpha) & =\sum_{2 \leq m \leq n} \frac{2}{m !}(-1)^{m}\left[\begin{array}{c}
m \\
2
\end{array}\right] \frac{m}{n}\left(\begin{array}{c}
2 n \\
n-m
\end{array}\right) z^{n} \\
& =2 \sum_{2 \leq m \leq n} H_{m-1}(-1)^{m} \frac{1}{n}\left(\begin{array}{c}
2 n \\
n-m
\end{array}\right) z^{n} \\
& =2 \sum_{1 \leq j<m \leq n} \frac{1}{j}(-1)^{m} \frac{1}{n}\left(\begin{array}{c}
2 n \\
n-m
\end{array}\right) z^{n} \\
& =2 \sum_{1 \leq j<n} \frac{1}{j}(-1)^{j-1} \frac{1}{n}\left(\begin{array}{c}
2 n-1 \\
n-j-1
\end{array}\right) z^{n} .
\end{aligned}
$$

In the last step we used the formula

$$
\sum_{j<m \leq n}(-1)^{m}\left(\begin{array}{c}
2 n \\
n-m
\end{array}\right)=(-1)^{j-1}\left(\begin{array}{c}
2 n-1 \\
n-j-1
\end{array}\right),
$$

which is a standard summation for binomial coefficients [3].

To obtain the form proposed by Knuth, we still need to prove that

$$
\left(\begin{array}{c}
2 n \\
n
\end{array}\right)\left(H_{2 n-1}-H_{n}\right)=2 \sum_{1 \leq j<n} \frac{(-1)^{j-1}}{j}\left(\begin{array}{c}
2 n-1 \\
n-j-1
\end{array}\right) .
$$

Modern computer algebra systems readily simplify the difference of these two sides to 0 , as expected.

\section{Connection with harmonic numbers - the general case}

In [4], there is the general formula

$$
\frac{1}{n !}\left[\begin{array}{c}
n+1 \\
r+1
\end{array}\right]=(-1)^{r} \sum_{\{r\}} \prod_{j=1}^{l} \frac{(-1)^{i_{j}}}{i_{j} !}\left(\frac{H_{n}^{\left(r_{j}\right)}}{r_{j}}\right)^{i_{j}} .
$$

Here, the sum is over all partitions of $r$ :

$$
r=i_{1} r_{1}+\cdots+i_{l} r_{l}
$$

with parts $r_{1}>\cdots>r_{l} \geq 1$ and positive integers $i_{1}, \ldots, i_{l}$. As an example, the partitions of $r=4$ are $4,3+1,2+2,2+1+1,1+1+1+1$, written alternatively as $1 \cdot 4,1 \cdot 3+1 \cdot 1$, $2 \cdot 2,1 \cdot 2+2 \cdot 1,4 \cdot 1$.

There appear higher order harmonic numbers as well:

$$
H_{n}^{(i)}=\sum_{1 \leq k \leq n} \frac{1}{k^{i}}
$$


Here are the first few instances:

$$
\begin{aligned}
\frac{1}{n !}\left[\begin{array}{c}
n+1 \\
2
\end{array}\right] & =H_{n}, \\
\frac{1}{n !}\left[\begin{array}{c}
n+1 \\
3
\end{array}\right] & =-\frac{1}{2} H_{n}^{(2)}+\frac{1}{2} H_{n}^{2} \\
\frac{1}{n !}\left[\begin{array}{c}
n+1 \\
4
\end{array}\right] & =\frac{1}{3} H_{n}^{(3)}-\frac{1}{2} H_{n}^{(2)} H_{n}+\frac{1}{6} H_{n}^{3}, \\
\frac{1}{n !}\left[\begin{array}{c}
n+1 \\
5
\end{array}\right] & =-\frac{1}{4} H_{n}^{(4)}+\frac{1}{3} H_{n}^{(3)} H_{n}+\frac{1}{8}\left(H_{n}^{(2)}\right)^{2}-\frac{1}{4} H_{n}^{(2)} H_{n}^{2}+\frac{1}{24} H_{n}^{4} .
\end{aligned}
$$

This allows to replace $\frac{1}{(m-1) !}\left[\begin{array}{l}m \\ p\end{array}\right]$ in

$$
(\log C(z))^{p}=\sum_{p \leq m \leq n} \frac{1}{(m-1) !}\left[\begin{array}{c}
m \\
p
\end{array}\right](-1)^{m-p} \frac{p !}{n}\left(\begin{array}{c}
2 n \\
n-m
\end{array}\right) z^{n}
$$

by an expression involving $H_{m-1}^{(1)}, \ldots, H_{m-1}^{(p-1)}$.

\section{Extension}

If instead of $u=z(1+u)^{2}$ we work with $u=z(1+u)^{\lambda}$, then we deal with the generating function of extended (generalized) Catalan numbers

$$
C_{\lambda}(z)=\sum_{n \geq 0}\left(\begin{array}{c}
1+n \lambda \\
n
\end{array}\right) \frac{z^{n}}{1+n \lambda}
$$

From [3], we infer that

$$
u^{m}=\sum_{n \geq m}\left(\begin{array}{c}
\lambda n+m \\
n
\end{array}\right) \frac{m}{\lambda n+m} z^{n}
$$

So

$$
\begin{aligned}
F(z, \alpha) & =\sum_{p \geq 0} \frac{\alpha^{p}}{p !}\left(\log C_{\lambda}(z)\right)^{p}=\exp \left(\alpha \log C_{\lambda}(z)\right)=C_{\lambda}^{\alpha}(z) \\
& =(1+u)^{\alpha}=\sum_{m \geq 0}\left(\begin{array}{c}
\alpha \\
m
\end{array}\right) u^{m} \\
& =\sum_{0 \leq k \leq m \leq n} \frac{1}{m !}(-1)^{m-k}\left[\begin{array}{c}
m \\
k
\end{array}\right] \alpha^{k}\left(\begin{array}{c}
\lambda n+m \\
n
\end{array}\right) \frac{m}{\lambda n+m} z^{n}
\end{aligned}
$$

The desired formula follows from reading off coefficients of $\alpha^{p}$ :

$$
\left(\log C_{\lambda}(z)\right)^{p}=p !\left[\alpha^{p}\right] F(z, \alpha)=\sum_{p \leq m \leq n} \frac{p !}{m !}(-1)^{m-p}\left[\begin{array}{c}
m \\
p
\end{array}\right]\left(\begin{array}{c}
\lambda n+m \\
n
\end{array}\right) \frac{m}{\lambda n+m} z^{n} .
$$




\section{References}

[1] W. Chu, Logarithms of a binomial series: extension of a series of Knuth, Math. Commun. 24 (2019), 83-90, https://www.mathos.unios.hr/mc/index.php/mc/article/ view/2878.

[2] N. G. de Bruijn, D. E. Knuth and S. O. Rice, The average height of planted plane trees, in: R. C. Read (ed.), Graph Theory and Computing, Academic Press, New York, pp. 15-22, 1972.

[3] R. L. Graham, D. E. Knuth and O. Patashnik, Concrete Mathematics, Addison-Wesley, Reading, Massachusetts, 2nd edition, 1994, https://www-cs-faculty.stanford.edu/ $\sim$ knuth/gkp.html.

[4] D. B. Grünberg, On asymptotics, Stirling numbers, gamma function and polylogs, Results Math. 49 (2006), 89-125, doi:10.1007/s00025-006-0211-7.

[5] D. E. Knuth, (3/2)-ary Trees (20th Annual Christmas Tree Lecture), 2014, https://www . youtube. com/watch?v=P 4AaGQIoOHY.

[6] D. E. Knuth, Problem 11832 (Problems and Solutions), Amer. Math. Monthly 122 (2015), 390, doi:10.4169/amer.math.monthly.122.04.390.

[7] D. E. Knuth, Log-squared of the Catalan generating function (Solution to Problem 11832), Amer. Math. Monthly 124 (2017), 660-661, doi:10.4169/amer.math.monthly.124.7.659.

[8] R. P. Stanley, Enumerative Combinatorics, Volume I, The Wadsworth \& Brooks/Cole Mathematics Series, Wadsworth \& Brooks/Cole, Monterey, California, 1986, doi:10.1007/ 978-1-4615-9763-6. 